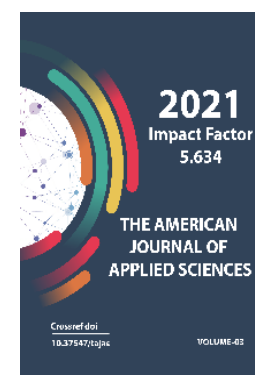

Journal Website: http://theamericanjour nals.com/index.php/taj as

Copyright: Original content from this work may be used under the terms of the creative commons attributes 4.0 licence.

\section{Electrometric Determination Of Binary Mixtures In Food}

\author{
Narbek Sapaevich Palvanov \\ Candidate Of Chemical Sciences, Docent Of Department "Natural Science", Urgench Branch \\ of The Tashkent Medical Academy, Uzbekistan
}

Mukhiddin Sultanovich Mekhmanov

Candidate Of Chemical Sciences, Head Of Department "Natural Science", Urgench Branch Of The Tashkent Medical Academy, Uzbekistan

\title{
ABSTRACT
}

In this paper, the possibility of acid-base titration of binary mixtures of dicarboxylic acids in mixed and non-aqueous solutions is investigated. The possibility of titrimetric potentiometric determination of binary mixtures of dicarboxylic acids is shown.

\section{KEYWORDS}

Electrometric, potentiometric titrating, oxalic acid, wine acid, titrating, succinic acid.

\section{INTRODUCTION}

Dicarboxylic acids are important in chemistry, chemical technology, medicine, pharmaceutical industry and other industries. An analytically important problem is the determination of mixtures of components of different nature. $[1,2]$.

Carboxylic acids in water are weak acids with close acidity constants; therefore, titrimetric determination of their mixtures in water is not possible. To resolve this issue, titration should be carried out in an environment of differentiating non-aqueous solvents. The correct choice of solvent is essential to the success of the analysis. Often, for acid-base titration, a solvent is selected based on the value of the auto-proteolysis constant. As indicated above (the work presented by us), the more, the more the acid strength is differentiated. The solvent we have chosen, isopropyl alcohol, has an auto-proteolysis constant of 20.80. Consequently, it can differentiate the strengths of some of the studied acids. 


\section{MATERIALS AND RESEARCH METHODS}

Sulfuric, oxalic, tartaric, succinic acids and their binary mixtures were chosen as objects. Mixtures of dicarboxylic acids were titrated with solutions of hydroxide, potassium isopropylate. So, propanol-2 and methyl ethyl ketone were used as solvents $[1,2,3,4]$.

The study of acid-base properties and potentiometric titration were carried out on a $\mathrm{pH}-340 \mathrm{pH}$ meter and similar modifications of $\mathrm{pH}$ meters. An EVL-1M3.1 silver chloride electrode filled with a saturated solution of potassium chloride in water or ethyl alcohol (when working in non-aqueous and mixed solutions) was used as a reference electrode. An ESL-43-07 glass hydrogen-selective electrode (for acid-base titration) and an EPV-1 platinum electrode (for oximetric titration) served as an indicator electrode.

Potentiometric titration is a method of qualitative and quantitative analysis based on measuring the potentials that arise between the test solution and an electrode immersed in it and is used to analyze transparent, colored and turbid solutions, as well as low concentrations of substances and for the quantitative analysis of various pharmacopeia preparations and other substances. Using it, it is possible to more objectively and reliably establish, therefore it has found wide practical application, especially in factory laboratories and in express analysis. Potentiometric titration is based on the determination of t.e. according to the results of potentiometric measurements, near which there is a sharp change (jump) in the potential of the indicator electrode. Such a jump is observed when the reaction components are direct participants in the electrode process. As is known, potentiometric titration by acid-base interaction is performed with a glass electrode, while a silver chloride electrode is used as a reference electrode. In potentiometric titrations, as in other methods of determination, the reactions of interaction of the components must proceed strictly stoichiometrically, at the same time have a high rate and, if possible, proceed completely and completely.

\section{RESEARCH RESULTS AND DISCUSSION}

In aqueous solutions, the acidity constants of the studied acids are close; therefore, the titrimetric determination of their mixtures, especially weak ones, is a difficult analytical problem. The values of the acidity constants of the studied acids in water are close (oxalic $\mathrm{pK}_{\mathrm{a}}^{\prime}=1,25$ and $\quad \mathrm{pK}_{\mathrm{a}}^{\prime \prime}=4,27$; tartaric $\mathrm{pK}_{\mathrm{a}}^{\prime}=3,04$ and $\mathrm{pK}_{\mathrm{a}}^{\prime \prime}=4,37$; succinic $\mathrm{pK}_{\mathrm{a}}^{\prime \prime}=4,37$; and, $\mathrm{pK}_{\mathrm{a}}^{\prime \prime}=5,48$ ), therefore, the analysis of their mixtures in an aqueous solution is not possible. such a condition is not feasible. $[4,5,9]$ To ensure the fulfillment of this condition, a change in the strength of acids is required by adding non-aqueous solvents to the water, the acidity scale of which would be as large as possible that in water or a complete replacement of water with an organic solvent with a larger scale $\Delta \mathrm{pK}_{\mathrm{a}}=\mathrm{pK}_{\mathrm{a}}^{1}-\mathrm{pK}_{\mathrm{a}}^{2} \geq 4$. As was shown earlier, the addition of individual polybasic acids of non-aqueous organic solvents to aqueous solutions led to an improvement in the conditions for their titration by dissociation steps. To confirm this, we chose isopropyl alcohol, which has an autoproteolysis constant of 20.80. m, it must differentiate the strength of the studied acids, 
which is confirmed by experiments. According to the data obtained, it can be noted that titration in an isopropyl alcohol medium makes it possible to determine with sufficient accuracy not only individual acids, but also their mixtures. $[3,5,6,7]$. As an example, Table
1 shows the results of titration of a mixture of oxalic - succinic and a mixture of mineral sulfuric acid with citric acid.

Results of potentiometric titration of binary mixtures of polybasic acids in propanol-2 $(\varepsilon=18.3)$.

Table 1.

\begin{tabular}{|c|c|c|c|c|c|c|c|c|}
\hline \multirow[t]{2}{*}{$\begin{array}{c}\text { Structure } \\
\text { mixes }\end{array}$} & \multicolumn{2}{|c|}{$\begin{array}{l}\text { Introduced, } \\
\text { mg }\end{array}$} & \multicolumn{2}{|c|}{ Found, mg } & \multicolumn{2}{|c|}{ S } & \multicolumn{2}{|c|}{$\mathrm{S}_{\mathrm{r}}$} \\
\hline & 1 & 2 & 1 & 2 & 1 & 2 & 1 & 2 \\
\hline 1 Sorrel & 34,88 & 73,53 & $34,82 \pm 0,34$ & $73,28 \pm 0,25$ & 0,32 & 0,24 & 0,009 & 0,003 \\
\hline \multirow[t]{2}{*}{ 2.Amber } & 34,54 & 36,54 & $34,49 \pm 0,31$ & $36,61 \pm 0,20$ & 0,29 & 0,19 & 0,008 & 0,006 \\
\hline & 17,44 & 73,08 & $17,41 \pm 0,45$ & $73,12 \pm 0,18$ & 0,43 & 0,17 & 0,025 & 0,002 \\
\hline 1.Sulfur & 3,00 & 9,60 & $2,98 \pm 0,18$ & $9,44 \pm 0,21$ & 0,17 & 0,20 & 0,058 & 0,021 \\
\hline \multirow[t]{2}{*}{ 2.Lemon } & 3,00 & 19,20 & $2,98 \pm 0,18$ & $19,02 \pm 0,39$ & 0,17 & 0,37 & 0,058 & 0,019 \\
\hline & 3,00 & 28,80 & $2,98 \pm 0,18$ & $28,30 \pm 1,10$ & 0,17 & 1,05 & 0,058 & 0,037 \\
\hline
\end{tabular}

The titration curves of binary mixtures of dicarboxylic acids show three distinct potential jumps (Fig. 1).

In a mixture of oxalic and succinic acids, the first jump corresponds to the titration of oxalic acid according to the first dissociation constant, the second to joint titration of oxalic acid to the second and succinic acid according to the first constant, and the third to titration of succinic acid to the second constant. In the case of titration of a mixture of sulfuric and citric acids by dissociation stages, sulfuric acid is titrated first, and then citric acid. $[5,6,7,8]$. 


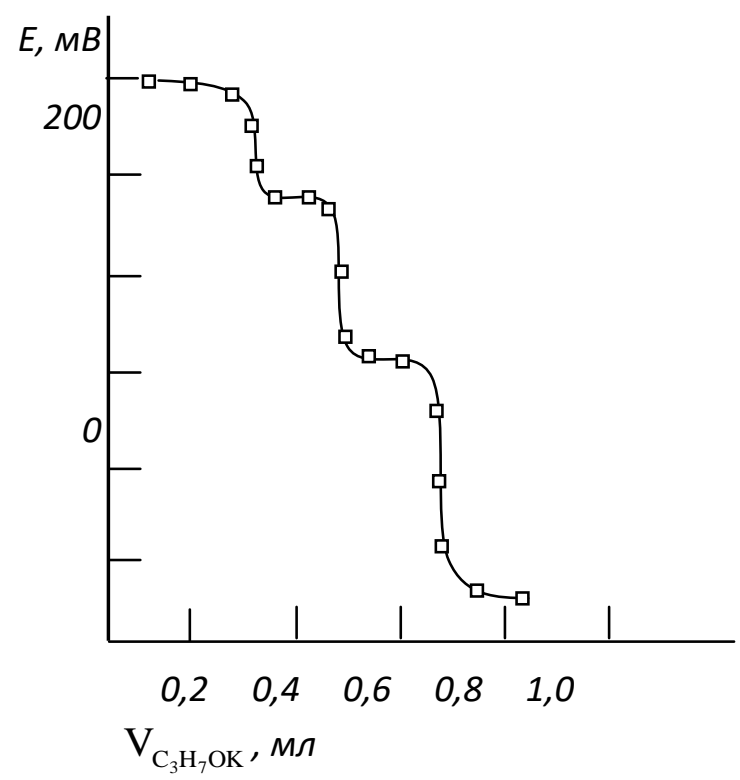

Fig. 1. Curves of potentiometric titration of a binary mixture of polybasic acids with potassium isopropyl in isopropanol.

To study the effect of the value of the autoproteolysis constant index, methyl ethyl ketone was added to isopropyl alcohol, having a volume ratio of $1: 1$. Both of these solvents have almost the same dielectric constant. $\left(\varepsilon_{\mathrm{C}_{3} \mathrm{H}_{7} \mathrm{OH}}=18,3\right.$ and $\left.\varepsilon_{\mathrm{CH}_{3} \mathrm{COC}_{2} \mathrm{H}_{5}}=18,4\right)$. In a mixed solvent medium - isopropyl alcohol methyl ethyl ketone (1: 1), binary mixtures of acids of different nature are titrated with a solution of potassium isopropyl ate: sulfuric oxalic, oxalic - citric. In a binary mixture of sulfuric-oxalic acid, sulfuric acid is first titrated according to the first jump, then sulfuric (according to the second constant) and oxalic (according to the first constant) acids are titrated together - according to the second jump, and then oxalic acid is titrated in the third jump (according to its second constant ). In the case of a binary mixture of acids (oxalic-citric, etc.), there are three potential jumps. The first of them corresponds to the titration of oxalic acid in the first stage, the second - to the joint titration of oxalic acid in the second and citric acid in the first and second stages, the third in citric acid in the third stage. $[5,7,8,9]$.

As the curves and the obtained results show, at the same values of the dielectric constants, the differentiating effect of the solvent is determined by the values of their autoproteolysis constants. In these considered examples, the values of the dielectric constants of the solvents were practically the same (18.3 and 18.4). Consequently, the determining factor of the differentiating effect of the solvent in the 
titration of mixtures of acids was the values of and MEK (20.8 and 25.85, respectively). the auto-proteolysis constants of isopropanol

\section{Results of potentiometric titration of binary mixtures of acids with a solution of $\mathrm{C}_{3} \mathrm{H}_{7} \mathrm{OK}$ in a} mixture of isopropyl alcohol - methyl ethyl ketone $(1: 1, \varepsilon=18,35)$

$$
(\mathrm{n}=6, \overline{\mathrm{x}} \pm \Delta \mathrm{x}, \mathrm{P}=0,95) \operatorname{Tab} 2 .
$$

\begin{tabular}{|c|c|c|c|c|c|c|c|c|}
\hline \multirow{2}{*}{$\begin{array}{c}\text { Structure } \\
\text { mixes }\end{array}$} & \multicolumn{2}{|c|}{$\begin{array}{l}\text { Introduced, } \\
\text { mg }\end{array}$} & \multicolumn{2}{|c|}{ Found, mg } & \multicolumn{2}{|c|}{ S } & \multicolumn{2}{|c|}{$\mathrm{S}_{\mathrm{r}}$} \\
\hline & 1 & 2 & 1 & 2 & 1 & 2 & 1 & 2 \\
\hline \multirow{4}{*}{$\begin{array}{l}\text { 1 Sorrel } \\
\text { 2.Amber }\end{array}$} & 3,00 & 4,50 & $3,01 \pm 0,05$ & $4,56 \pm 0,03$ & 0,05 & 0,03 & 0,016 & 0,006 \\
\hline & 3,00 & 9,00 & $3,01 \pm 0,02$ & $9,03 \pm 0,12$ & 0,02 & 0,02 & 0,006 & 0,013 \\
\hline & 3,00 & 3,50 & $3,01 \pm 0,01$ & $13,70 \pm 0,29$ & 0,01 & 0,28 & 0,003 & 0,021 \\
\hline & 3,00 & 18,00 & $3,01 \pm 0,02$ & $18,00 \pm 0,36$ & 0,02 & 0,34 & 0,006 & 0,019 \\
\hline \multirow{3}{*}{$\begin{array}{l}\text { 1.Sulfur } \\
\text { 2.Lemon }\end{array}$} & 9,60 & 4,50 & $9,55 \pm 0,05$ & $4,52 \pm 0,10$ & 0,05 & 0,09 & 0,005 & 0,021 \\
\hline & 4,80 & 9,0 & $4,78 \pm 0,03$ & $8,97 \pm 0,16$ & 0,03 & 0,15 & 0,006 & 0,016 \\
\hline & 9,60 & 9,0 & $9,62 \pm 0,12$ & $8,95 \pm 0,21$ & 0,11 & 0,20 & 0,012 & 0,022 \\
\hline
\end{tabular}

The results of titration of binary mixtures of acids in a mixture of isopropyl alcohol - methyl ethyl ketone (1: 1) are given in table. 2 in which the dielectric constant of the medium practically did not change. As can be seen from the data in Table 2, the results obtained are quite satisfactory, which can be seen from the values that do not exceed 0.022 (for a binary mixture)

\section{CONCLUSIONS}

1. Thus, our study has established that the addition of a solvent with a low dielectric constant improves the titration conditions, which is associated with a decrease in the total dielectric constant and the autoproteolysis constant of the solvent.

2. A relationship has been established between the constant of acidity of substances and the constant of autoproteolysis and the dielectric constant of the medium. It is shown that an increase in the auto-proteolysis constant and a decrease in the dielectric constant lead to a decrease in the strength of dissolved carboxylic acids and, consequently, to an increase in the differentiating effect of the solvent.

3. The choice of solvents for acid-base titration of carboxylic, mineral and amino acids in their mixtures has been substantiated and experimentally 
confirmed. The leveling-differentiating effect of solvent mixtures of different nature and composition in relation to the substances under study has been studied.
9. Denesh I. Titration in non-aqueous media. M .: Mir. 1971.

\section{REFERENCES}

1. Kreshkov A.P. Analytical chemistry of non-aqueous solutions. - $M$.: Chemistry, 1980 . -256 p.

2. Kreshkov A.P., Aldarova N.Sh., Smolova N.T., Turovtseva G.V. // J. analyte. chemistry. - 1970. - T. 25. - No. 7. - pp. 1392-1397.

3. Khudyakova T.A., Arbatsky A.P. Acidbase properties of electrolytes and criteria for their analysis. - $M$.: Chemistry, 1988 . -62 p.

4. Mchedlov-Petrosyan N.O. Differentiation of the strength of organic acids in true and organized solutions // J. Analyt. chemistry. - 2006. - T. 61. - No. 3. - pp. 329-330.

5. Kreshkov A.P. Analytical chemistry of non-aqueous solutions. $-\mathrm{M} .:$ Chemistry, 1982. - $256 \mathrm{p}$.

6. Fayzullaev O., Fayzullaev O.O. Titrimetric determination of lead // Actual problems of analytical chemistry: Abstracts. report All-Russian. conf. -M .: 2002. - pp. 32-33.

7. Palvonov NS Electrometric methods for the determination of some carboxylic and aprotic acids in aqueous and mixed solutions. Dis .... cand. chem. sciences. T., 2012. - pp.72-76.

8. Tugizov M.Y., Fayzullaev O.F., Mukhammadiev N.Q. Appeciation of acid-base properties of cobalt compounds // Anal. Chem. and Applied Spectroscopy / A Global technical conference: Abstracts. -New Orleans, LA: 2002. - P. 1539. 